\title{
Analysis of Blends of Cashew Exudate and Poly (Vinyl Chloride)
}

\author{
Alisi Ikechukwu Ogadimma ${ }^{1,}$, , Ngele Sylvester Okechukwu ${ }^{2}$, Uduma Anya Uduma ${ }^{1}$ \\ ${ }^{1}$ Department of Applied Chemistry, Federal University, Dutsinma, Nigeria \\ ${ }^{2}$ Department of Industrial Chemistry, Ebonyi State University, Abakaliki, Nigeria
}

Email address:

ialisi@fudutsinma.edu.ng (A. I. Ogadimma), ikeogadialisi@gmail.com (A. I. Ogadimma)

${ }^{*}$ Corresponding author

\section{To cite this article:}

Alisi Ikechukwu Ogadimma, Ngele Sylvester Okechukwu, Uduma Anya Uduma. Analysis of Blends of Cashew Exudate and Poly (Vinyl Chloride). American Journal of Polymer Science and Technology. Vol. 3, No. 2, 2017, pp. 21-28. doi: 10.11648/j.ajpst.20170302.11

Received: July 29, 2016; Accepted: February 6, 2017; Published: April 26, 2017

\begin{abstract}
In this research the mechanical and sorption properties of purified Anacardium Occidentale L. exudate and poly(vinyl chloride) blends were investigated. The purified Cashew Gum PCG and PVC were compounded through melt blending technique followed by compression moulding on a Carver Laboratory Press set at $150^{\circ} \mathrm{C}$. The blended samples were then subjected to tensile, hardness, impact and sorption tests. The results of the mechanical tests indicate a general decrease in Young's modulus, tensile strength and percentage elongation with increasing composition of the purified exudate in the blended samples. There was an improvement in hardness properties with increase in exudate composition with a maximum value of $69.3 \mathrm{HRF}$ for the composition $40 \% \mathrm{PVC} / 60 \% \mathrm{PCG}$. For the Charpy impact tests, the maximum and minimum values recorded were $10227.27 \mathrm{Jm}^{2}$ and $6818.18 \mathrm{Jm}^{-2}$ respectively. The absorption test results indicate swelling in aqueous media which increases as the composition of PCG increases. This swelling behaviour of the blended samples in aqueous media indicate their susceptibility to hydrolytic degradation, and this can serve as a means of controlling environmental pollution due to discarded plastic materials.
\end{abstract}

Keywords: Blended Samples, Mechanical Properties, Poly (Vinyl Chloride), Purified Cashew Gum, Sorption Properties

\section{Introduction}

Polymer blends are physical mixtures of two or more polymers with/without any chemical bonding between them. Polymers are increasingly used in many applications in view of their good strength and low densities. Blending of polymers for property improvement or for economic advantage has gained considerable importance in the field of polymer science in the last decade (George et al., 1986). Polymer blending provides a powerful route to engineering new properties in materials using available polymers. From Polymer blending it is possible to produce a range of materials with properties that are superior to that of each individual component polymers (Rhooet al., 1997; Oh and Kim, 1999; Pielichowski, 1999; Stephen et al., 2000; Tang and Liau, 2000; Pielichowski and Hamerton, 2000).

The use of polymers such as plastics have also come with some shortcomings as they resist degradation in natural conditions. Thus they cause waste disposal problems and release $\mathrm{CO}_{2}$ and dioxins during combustion, which cause pollution and global warming (Huang, 1995).

In recent time, research is focusing increasingly on the development of polymers that combine the desired functionality during use and rapid degradation after disposal as a viable alternative to conventional nondegradable polymers, mostly for applications in which long degradation times are undesirable. Biodegradable polymers fit this context perfectly, since they degrade rapidly and contain nontoxic end products which have low permanence in the environment and are completely metabolized by soil microorganisms (Scott and Gillead, 1995).

The biopolymer of choice in this work is Anacardium occidentale exudate, popularly called Cashew tree gum. Cashew gum (CG) is an exudate polysaccharide from Anacardium occidentale trees. The plant is native to Brazil and grows in many tropical and sub-tropical countries. Major cashew growing areas in Nigeria are most states in the SouthEast and South-West (Ezeagu, 2002). Cashew gum has been 
studied widely for various pharmaceutical applications as it is inexpensive, non-toxic, biodegradable, and possesses appropriate physicochemical characteristics (Gyedu-Akotoet al., 2008 and Kumar et al., 2009).

PVC is a valuable thermoplastic with useful molecular structure and morphology due to its low price, good process ability, chemical resistance and low flammability (Mediha, etal., 2008). Hence, Poly(vinyl chloride) is extensively used for a broad range of applications.

In this present work, Cashew tree gum which was subjected to chemical purification was blended with poly(vinyl chloride), PVC of laboratory grade. These blends were prepared through melt mixing technique using the tworoll mill maintained at appropriate processing conditions. This was followed by compression molding of the blended samples using a mould engraved with standard sample dimensions. The mechanical properties as well as the chemical resistance of the resulting PVC/PCG blends were investigated.

\section{Experimental}

\subsection{Materials and Equipment}

A list of all the materials and equipment used in this research work are listed in table 1 and 2 respectively.

Table 1. List of materials and their corresponding grades and source.

\begin{tabular}{lll}
\hline Material & Grade & Source \\
\hline $\begin{array}{l}\text { Poly(vinylchloride), } \\
\text { PVC (powder) }\end{array}$ & $\begin{array}{l}\text { K Value: } 65 \text {; Density: } \\
1.37 \mathrm{~g} / \mathrm{cm}^{3}\end{array}$ & BDH \\
Anacardium & & Plantation garden in \\
occidentale gum & Natural exudates & $\begin{array}{l}\text { Ahmadu Bello } \\
\text { University Zaria }\end{array}$ \\
Ethanol & & Aldrich \\
Benzene & Analytical & Aldrich \\
Chloroform & Analytical & Aldrich \\
Carbon Tetrachloride & Analytical & Aldrich \\
Cyclohexane & Analytical & Aldrich \\
Cyclohexanone & Analytical & Aldrich \\
Petroleum Ether & Analytical & Aldrich \\
Tetrahydrofuran & Analytical & Aldrich \\
Toluene & Analytical & Aldrich \\
1, 4-Dioxane & Analytical & Aldrich \\
\hline
\end{tabular}

Table 2. List of equipment used and their corresponding models.

\begin{tabular}{ll}
\hline Equipment & Model \\
\hline General Laboratory Centrifuge & SORVIAL75066180 \\
pH meter & Jenway 3505 \\
Infrared spectrophotometer & Shimadzu FTIR-8400S \\
Two-roll mill & 5183 \\
Tensometer & Hounsfield \\
Carver Hand press & $3851-0$ \\
Charpy Impact Tester & Cat. Nr.412 \\
Indentec Universal Hardness Testing & $8187.5 \mathrm{~L} \mathrm{Kv} \mathrm{model} \mathrm{B}$ \\
Machine & \\
\hline
\end{tabular}

\subsection{Purification of Cashew Gum Exudate}

Hard caked crude natural exudates of cashew gum were obtained with the aid of a knife from the bark of cashew trees located in a plantation garden in Samaru Campus of Ahmadu
Bello University Zaria. The cashew gum was purified according to the method described by Lima et al., 2002. Based on this method, the dried light colored cashew gum grade were manually sorted and further processed by milling in a domestic blender into particles small enough to pass through a $2.5 \mathrm{~mm}$ mesh-size sieve, and designated as crude cashew gum (CCG). The dried CCG gum was hydrated in a minimum amount of distilled water for a period of 24 hours. The resulting viscous solution was filtered by passage through a muslin cloth contained in a filter funnel, and subsequently subjected to centrifugation using a General Laboratory Centrifuge (GLC) at the rate of 450 r.p.m. for 10 min. As shown in table 1, various chemical reagents were employed to determine the best precipitating agent for CCG. The CCG solution was precipitated using ethanol of $96 \%$ conc. at room temperature and washed using petroleum ether. The dried purified gum was milled and screened through $180 \mu \mathrm{m}$ stainless steel sieve and the undersized fractions stored in air - tight powder bottles and labeled purified cashew gum (PCG).

\subsection{Procedure for Blend Formulation}

Each PVC and PCG powder was thoroughly mixed together in the two-roll mill to give blends of various compositions. $30 \mathrm{~g}$ of plastic/gum blend composition was formulated. The various compositions of the blend samples of PVC/PCG were obtained as shown in table 3.

Table 3. PCG/PVC blends composition.

\begin{tabular}{ll}
\hline Polymer & Gum (Weight \% Ratio) \\
\hline 100 & 0 \\
90 & 10 \\
80 & 20 \\
70 & 30 \\
60 & 40 \\
50 & 50 \\
40 & 60 \\
30 & 70 \\
20 & 80 \\
10 & 90 \\
\hline
\end{tabular}

The results of 100: 0 for PVC/PCG homopolymers were used as control. The blending technique employed is melt blending. A laboratory-scale two-roll mill was employed for blend preparations. The samples were blended with the front roll maintained at a temperature of $150^{\circ} \mathrm{C}$ while the rear roll was at temperature of $120^{\circ} \mathrm{C}$.

The PVC homopolymer was initially placed on the front roll for $3 \mathrm{~min}$ to facilitate processing. Then the roll mill was started and the PCG powder added intermittently a little at a time. The milling was continued for 5 minutes. Occasionally, the molten blend was scraped out from the roll, wrapped several times and milled back to the roll. After 5 min of milling, the molten blend was scraped out and cut into specimens of approximately $2 \mathrm{~cm} \times 2 \mathrm{~cm}$ in size. These pieces were then put into a grinding mill, and small pelletsized samples were obtained. The blended sample pellets were subjected to compression moulding at standard 
processing conditions and characterized by various analytical methods such as: absorption tests, tensile tests, impact tests and hardness tests.

\subsection{Compression Moulding}

The polymer blends were introduced into an aluminum mould sprayed with a mould release agent and wrapped with aluminum foil (for easy removal of the specimen after molding). This mold was designed to accommodate samples with dimensions according to ASTM standard. The compression molding was carried out on a Carver Laboratory Press equipped with temperature controllers. The temperature was set at $150^{\circ} \mathrm{C}$ at a pressure of 8.5 Tons for a determined period of 12 minutes. The molded PVC/PCG sample blends were then separated from the mold and labeled appropriately.

\subsection{Blend Characterization}

\subsubsection{Determination of the Density of Blended Samples}

The dimensions (length, width and thickness) of the blended polymer samples were accurately measured with the aid of venier calipers while their masses were measured using an electronic weighing balance. For each sample composition, three measurements were conducted and employed in the computation of the mean and standard deviation values. The above measurements were carried out in order to obtain the density of each blend composition. It is also aimed at determining the effect of PCG on the density of PVC. The densities of the blended samples were then compared with that of the control sample.

\subsubsection{Absorption Test}

Water absorption tests for the blend samples were investigated according to ASTM D0570 standard. The polymer blends were suspended in $200 \mathrm{ml}$ of distilled water at room temperature in desiccators for a total period of 7 days with readings taken after every 24 hours. After the required amount of time the samples were removed and gently dried using a filter paper to remove water adhering to its surface.. For each sample composition, three measurements were conducted and employed in the computation of the mean and standard deviation values. The degree of swelling (DS) was calculated using the expression:

$$
\mathrm{DS}=\left(\frac{\mathrm{m}_{2}-\mathrm{m}_{1}}{\mathrm{~m}_{1}}\right) \times 100
$$

Where, $\mathrm{m}_{1}$ and $\mathrm{m}_{2}$ are the initial and final masses of the sample blends respectively.

\subsubsection{Mechanical Tests}

\section{i. Tensile Test}

The tensile strength of the blended samples were investigated according to ASTM D3039 standard. Sample dimensions of: $100 \mathrm{~mm} \times 10 \mathrm{~mm} \times 4 \mathrm{~mm}$ length, breadth and thickness were used. The experiments were conducted on a universal loading machine type Hounsfield Tensometer of maximum capacity $20.00 \mathrm{KN}$ at room temperature. A guage length of $30 \mathrm{~mm}$ was used. Tensile analysis of the samples was accomplished using a tensile load of $2.00 \mathrm{KN}$ at moderate strain rate until the point of failure.. For each sample composition, three measurements were conducted and employed in the computation of the mean and standard deviation values. From the tensile analysis, force and elongation values were recorded. Young's modulus, ultimate tensile strength and percentage elongation were calculated from the resultant stress-strain curves.

\section{ii. Hardness Test}

Hardness test results of the blended samples were determined according to the standard ASTM D2240-89 using Indentec Hardness Testing Machine. Rockwell test (F-Scale) with 1/16 inch (steel ball) indentor was used. The minor load used was 10 $\mathrm{kg}$ while the major load used was $60 \mathrm{~kg}$ with an exposure time of 10 seconds. The hardness test on each of the blended samples was conducted at three different points distributed over the test piece to obtain mean values. These results were employed in the calculation of the standard deviation.

\section{iii. Charpy Impact Test}

Charpy impact test analysis for the samples was conducted according to ASTM 370 standard using a Charpy Impact Testing Machine of hammer capacity $15 \mathrm{~J}$ and $25 \mathrm{~J}$ respectively. In this analysis the $15 \mathrm{~J}$ capacity hammer was employed to determine the fracture energy of each sample. The impact strength of each sample was calculated using the expression:

$$
\text { Impact Strength }=\frac{\text { FractureEnergyRequired }}{\text { AreaoftheSample }}
$$

\section{Results and Discussions}

\subsection{Cashew Gum Purification Results}

During the process of gum purification, we observed that the best condition of hydration was in the ratio of $10 \mathrm{~g}$ of CCG in $20 \mathrm{ml}$ of distilled water. At the end of centrifugation, no insoluble particles were found at the bottom of the tubes. This clearly indicates that the first filtration process was highly successful in removing insoluble particles and extraneous materials from the gum. Ethanol of $96 \%$ conc. was observed to be the best precipitating agent for CCG at room temperature. When five different samples of $\mathrm{CCG}$ solutions were each precipitated with $300 \mathrm{ml}$ of ethanol, the mass of dry precipitates of PCG produced are giving in table 4.

Table 4. Mass of PCG produced by equal volumes of ethanol on a fixed volume of different samples of CCG solution.

\begin{tabular}{llll}
\hline $\begin{array}{l}\text { Sample } \\
\text { Code }\end{array}$ & $\begin{array}{l}\text { Vol. of } \\
\text { CCG }(\mathbf{m l})\end{array}$ & $\begin{array}{l}\text { Volume of } \\
\text { Ethanol }(\mathbf{m l})\end{array}$ & $\begin{array}{l}\text { Mass of Precipitate } \\
(\mathrm{g})\end{array}$ \\
\hline CCG301 & 100 & 300 & 45.00 \\
CCG302 & 100 & 300 & 45.04 \\
CCG303 & 100 & 300 & 45.34 \\
CCG304 & 100 & 300 & 45.70 \\
CCG305 & 100 & 300 & 45.76 \\
\hline
\end{tabular}


From table 4, the mass of precipitates obtained from CCG301 to CCG305 are $45.00 \mathrm{~g}, 45.04 \mathrm{~g}, 45.34 \mathrm{~g}, 45.70 \mathrm{~g}$, and $45.76 \mathrm{~g}$ respectively. From the above result the average mass of dry ppt of PCG obtained when $100 \mathrm{ml}$ of CCG solution is completely precipitated with $300 \mathrm{ml}$ of ethanol is $45.368 \mathrm{~g}$.

\subsection{Blend Formulation, Density Analysis and Water Sorption Tests}

The results of blend formulation indicate that sample blends with PCG composition higher than $60 \%$ were not successfully blended to give a rigid structure. Sample densities of PVC/PCG blends are graphically illustrated in fig. 1 with error bars denoting standard deviation. From fig. 1 , we observe that an increase in PCG composition increases the density of the resulting PVC/PCG sample blends. This increase is attributed to higher density. Also, the results of water sorption tests conducted on PVC/PCG blend samples are given in figure 2 with error bars denoting standard deviation.

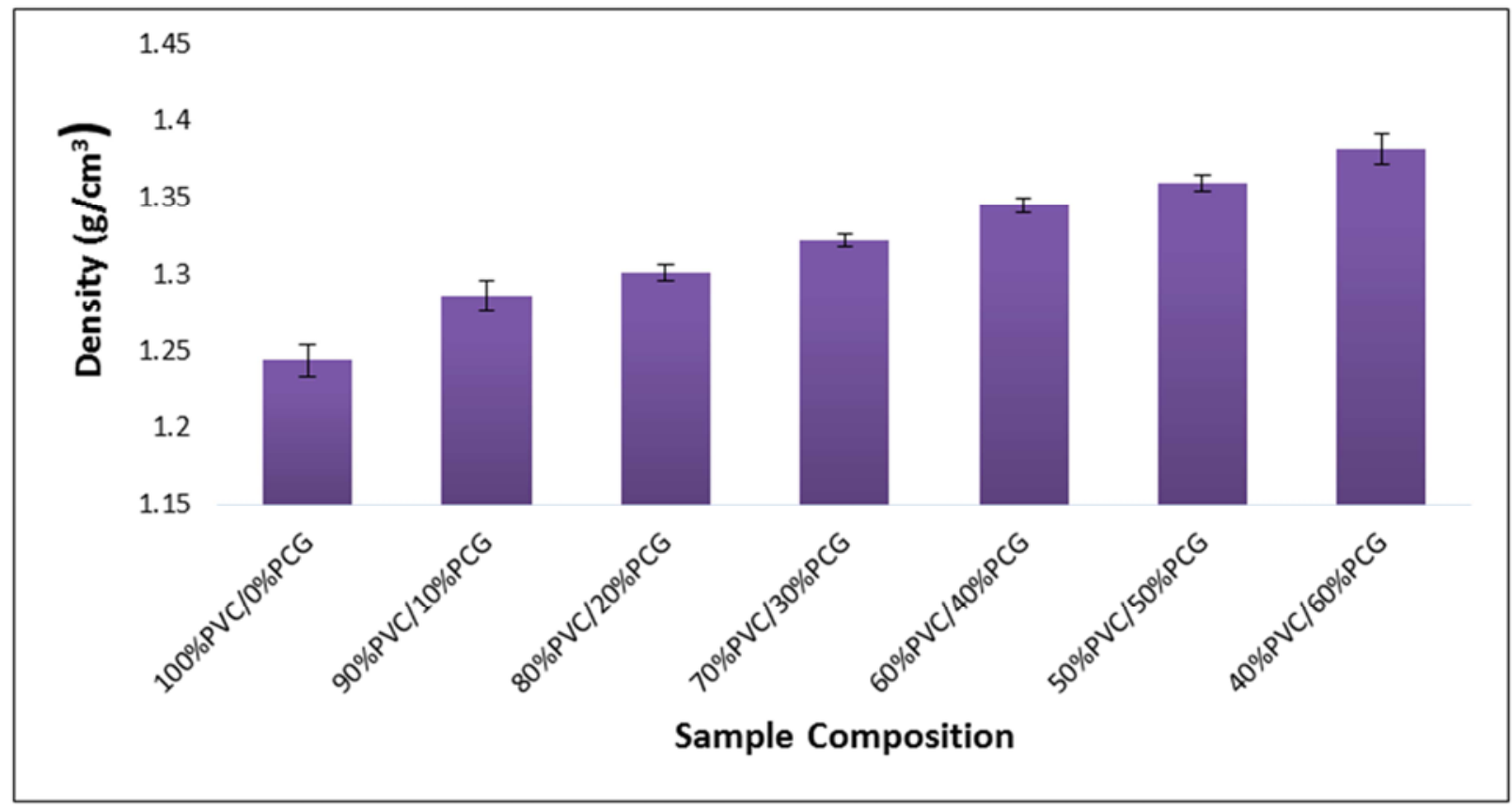

Fig. 1. Graphical representation of PVC/PCG sample blend densities against their corresponding compositions.

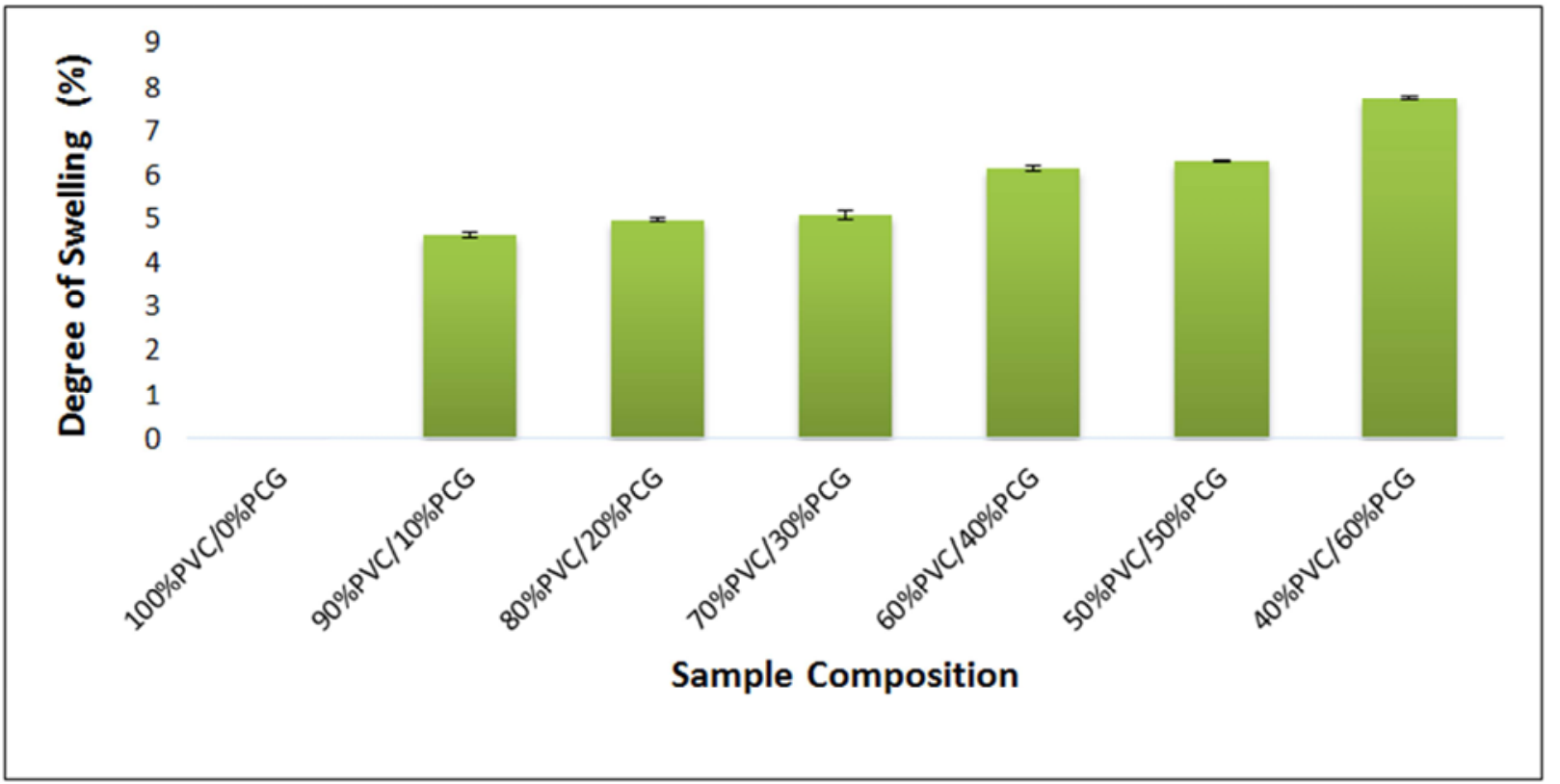

Fig. 2. $D S$ values for various compositions of $P V C / P C G$ blends.

Swelling is observed in the sample when it absorbs water which results to an increase in total volume. The PVC control sample did not exhibit welling behaviour throughout the period of the experiment as indicated by the zero percentage 
value. This is attributed to the passive nature of PVC to aqueous media at ambient conditions.

An examination of the swelling behaviour exhibited by the PVC/PCG blends show that the sample with composition $40 \% \mathrm{PVC} / 60 \% \mathrm{PCG}$ recorded the highest value of $7.75 \%$. This increase is in direct proportion to increase in PCG component in the blended samples. Thus the blending of PVC with PCG induces swelling behaviour into PVC

\subsection{Mechanical Tests}

\subsubsection{Tensile Test}

The results of tensile tests conducted on PVC/PCG blends and the control sample are shown in figure 3,4 and 5. All the samples used for analysis are of the same dimension with:

Area $\left(A_{o}\right): 10 \mathrm{~mm} \times 4 \mathrm{~mm}=40 \mathrm{~mm}^{2}=4.00 \times 10^{-5} \mathrm{~m}^{2}$

Gauge Length $\left(\mathrm{L}_{\mathrm{o}}\right): 30 \mathrm{~mm}$.

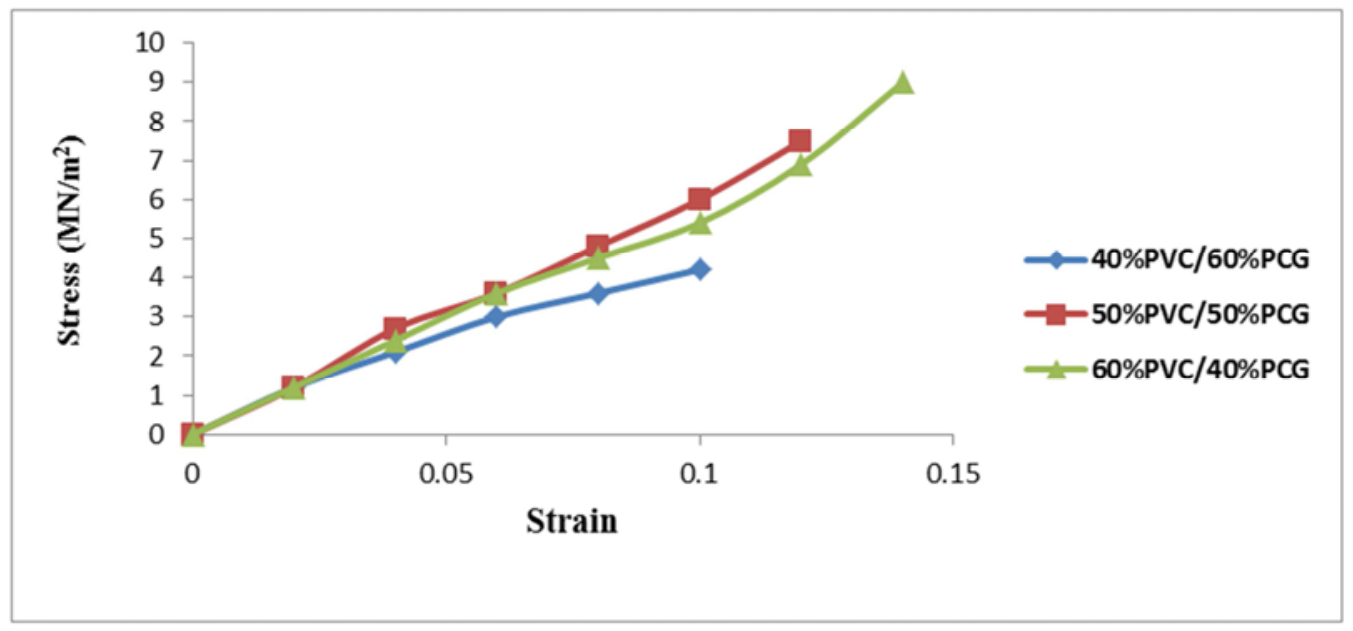

Fig. 3. Stress-strain curve of $40 \% P V C / 60 \% P C G$, $50 \% P V C / 50 \% P C G$ and $60 \% P V C / 40 \% P C G$.

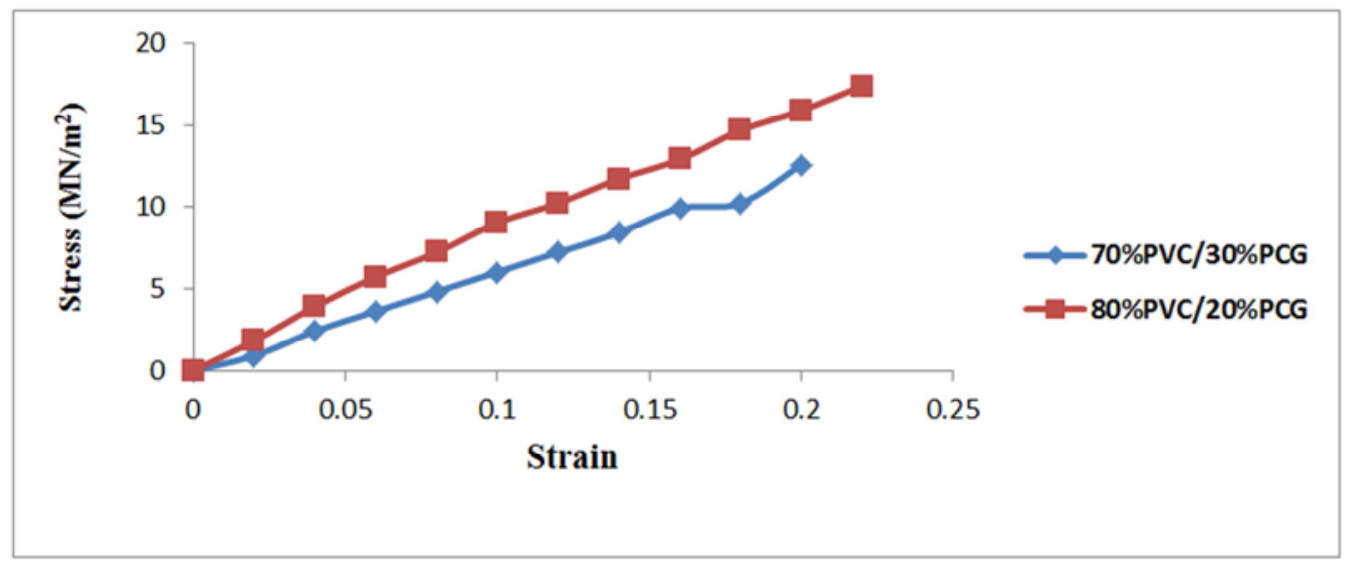

Fig. 4. Stress-strain curve of $70 \% P V C / 30 \% P C G$ and $80 \% P V C / 20 \% P C G$.

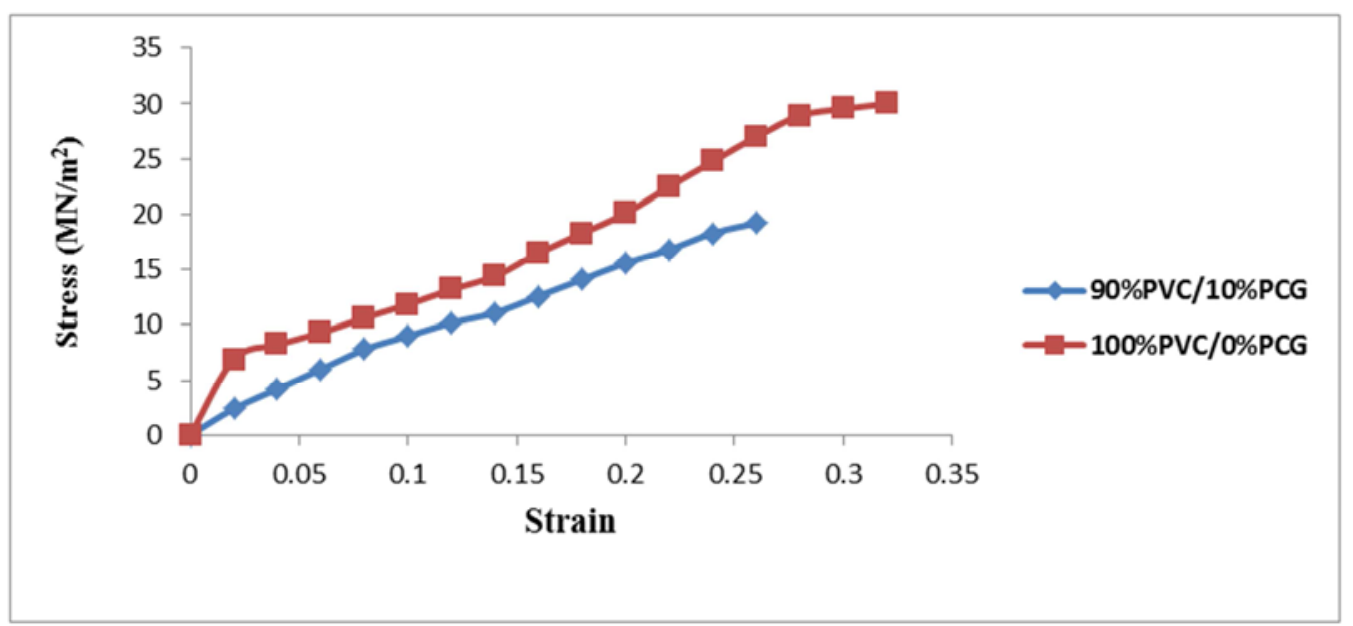

Fig. 5. Stress-strain curve of $90 \% P V C / 10 \% P C G$ and $100 \% P V C / 0 \% P C G$. 


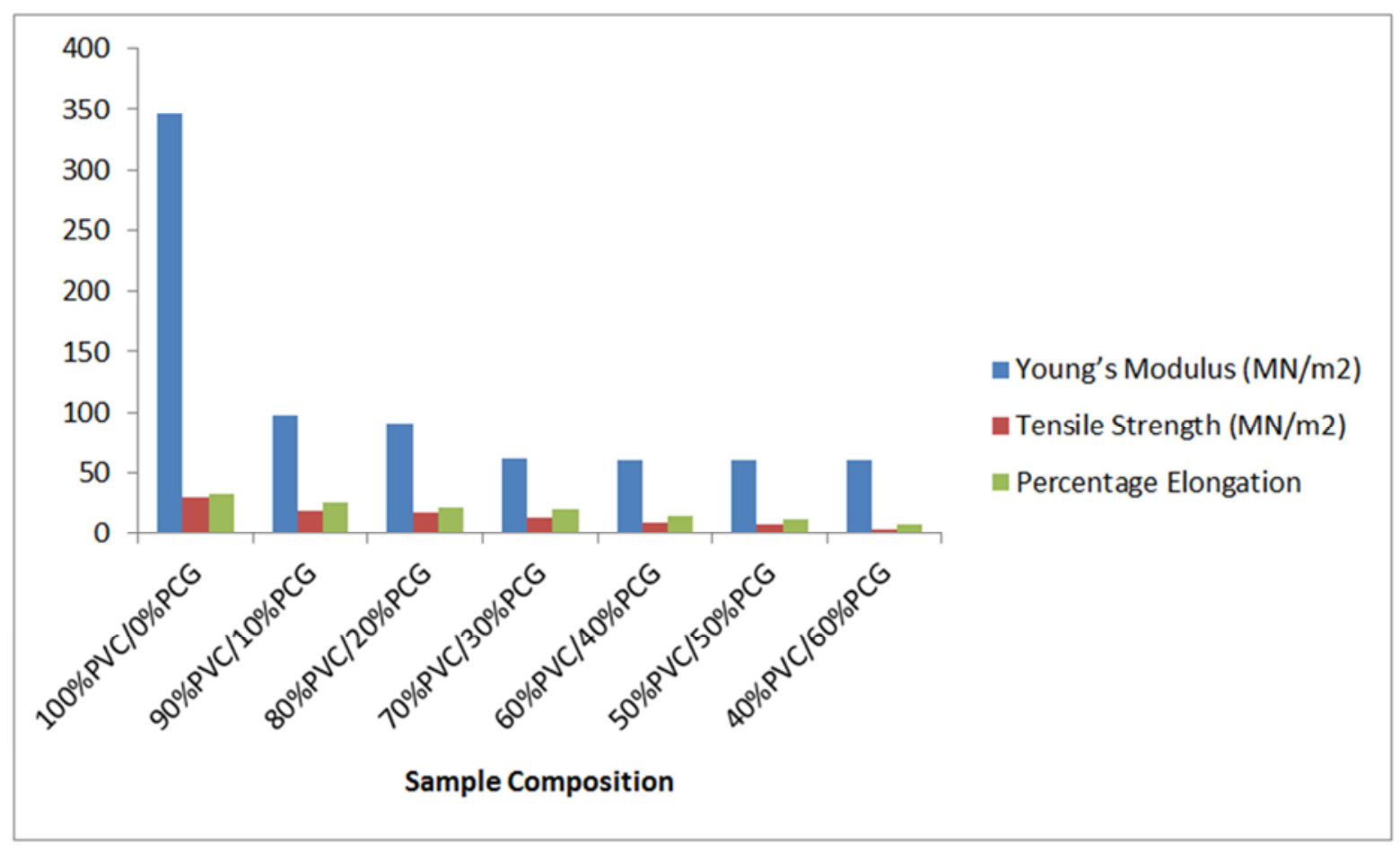

Fig. 6. Plot of Young's Modulus, Tensile Strength and Percentage Elongation of PVC/PCG Blend Samples.

The summary of the results of tensile properties as shown in figure 6, indicate a decrease in Young's modulus, tensile strength and percentage elongation of $\mathrm{PVC} / \mathrm{PCG}$ blended samples as the percentage of PCG in the blend composition increases. The general decrease in tensile properties of PVC/PCG blended samples in comparison to those of the control sample can be attributed to lower intermolecular interactions between PCG and PVC. Also, the general decrease in percentage elongations with increase in PCG concentrations is due to the low toughness of PCG as a result of its high brittleness.

\subsubsection{Hardness Test Analysis of Blended Samples}

The hardness test result of PVC blended samples are shown in figure 7 with error bars denoting standard deviation. These results were obtained using the Hardness Rockwell F - scale (HRF) and indicate an increase in hardness values as the proportion of PCG in the blend increases which is attributed to the good hardness properties of pure CG.

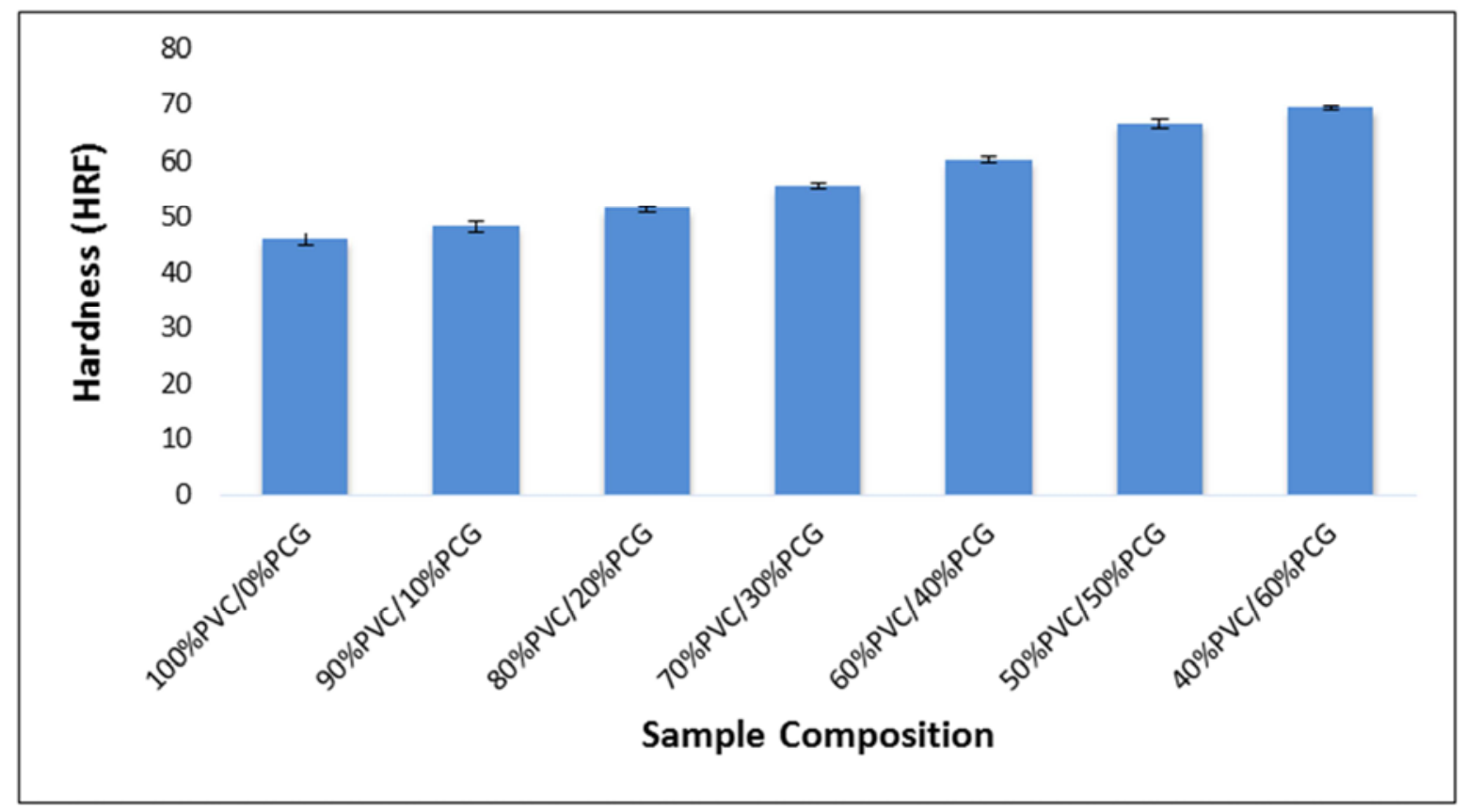

Fig. 7. Plot of hardness (HRF) against sample composition of $P V C / P C G$ blend samples. 


\subsubsection{Charpy Impact Test Analysis of Blended Samples}

The impact test results for PVC/PCG blended samples are given in figure 8 with error bars denoting standard deviation.

From this result, we observe that the impact strength of $\mathrm{PVC} / \mathrm{PCG}$ blended samples decrease with increase in the
PCG composition. The low impact strength values of samples with high proportions of PCG can be attributed to the phenomenon of plasticization as the PCG softens the polymer at high concentration values. This gives rise to low toughness of the resulting polymer blend.

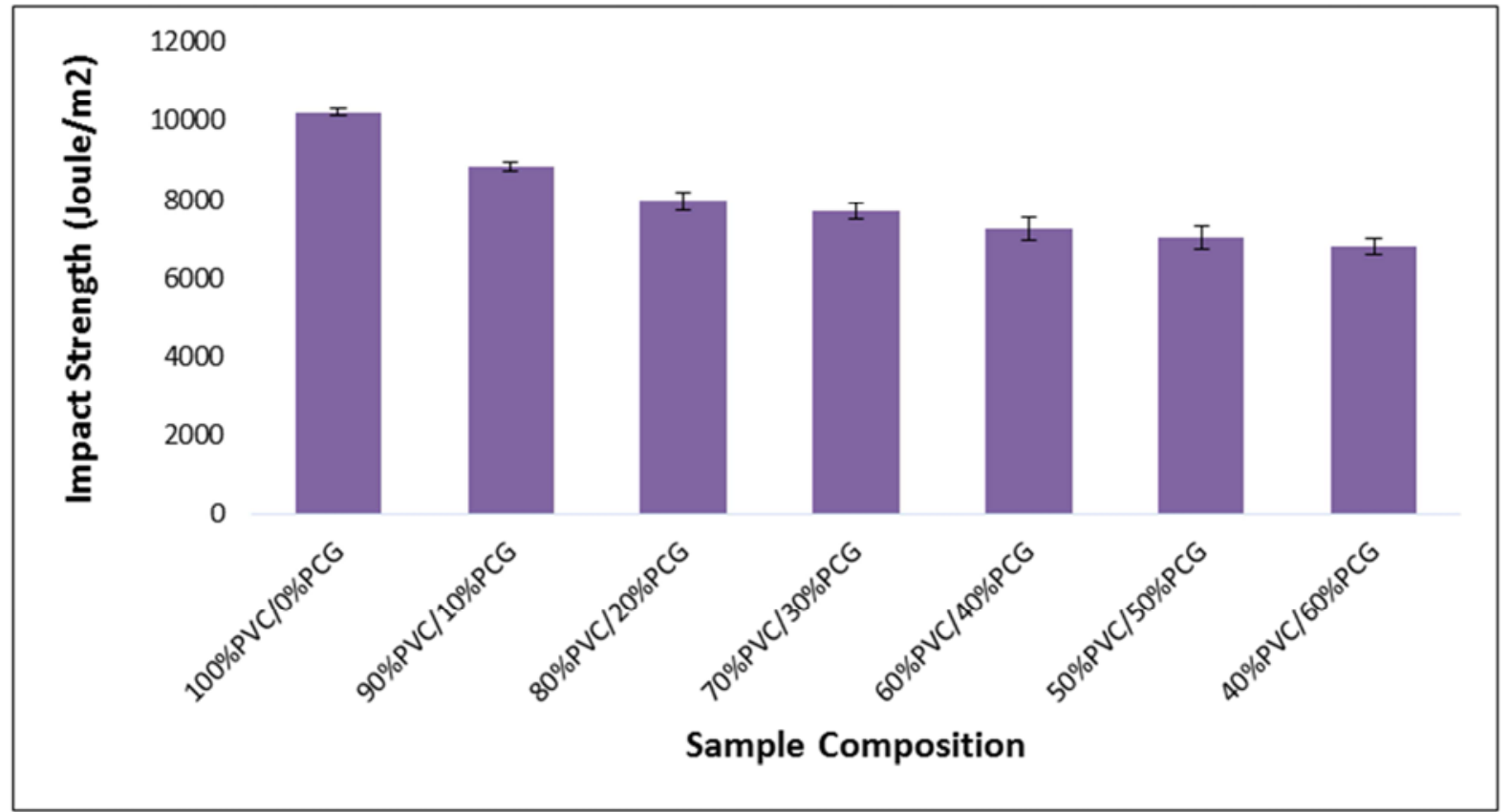

Fig. 8. Plot of impact strength against sample composition for PVC/PCG sample blends.

\section{Conclusion}

This research on the analysis of blends of cashew exudate and poly(vinyl chloride) shows that the maximum percentage of PCG needed to form a thermoplastic blend with PVC is $60 \%$.From this wok centered on cashew gum and the corresponding blends with $\mathrm{PVC}$, the following conclusions can be drawn:

The density values of the blended polymer samples were observed to increase in the presence of higher compositions of PCG in the blended samples. The higher density values are due to the higher density value of PCG.

The sorption properties of PVC/PCG blended samples indicated a gradual increase in DS values with increase in the percentage of PCG in the blended samples. This increase has been attributed to the high affinity of PCG to water.

The tensile properties of PVC such as Young's modulus, tensile strength and percentage elongation were generally observed to decrease with increase in the composition of PCG in the sample blends. These results generally indicate very weak intermolecular bonding forces between the PCG and thermoplastic molecules. The low percentage elongation of the resulting polymer blends is attributed to the fact that PCG is highly glassy at ambient conditions in the absence of moisture compared to the pure polymer resin.

The hardness properties of PVC blends indicate an improvement in hardness with increase in the composition of PCG to give a maximum value of $69.3 \mathrm{HRF}$ corresponding to the sample with composition $40 \% \mathrm{PVC} / 60 \% \mathrm{PCG}$. This general increase in hardness with increase in PCG concentration has been attributed to the good hardness properties of pure CG.

This work has also indicated that blending PVC with PCG lowers the impact strength of the thermoplastic. The lowering effect was observed to be higher at higher concentrations of PCG. This lowering effect has been linked to the phenomenon of plasticization in the resulting polymer blend.

From this research, PVC has successfully been blended with PCG. The blended polymer samples were observed to exhibit swelling behaviour in the presence of aqueous media more readily than the pure polymer sample. This swelling behaviour indicates a possible tendency for blended PVC to undergo hydrolytic degradation. This is a possible means of controlling environmental pollution as the blended polymer samples easily degrade when discarded into the environment after use. Also blending PVC with PCG has the tendency to lower the production cost of the PVC plastics as CCG is readily available in our immediate environment.

\section{References}

[1] Ezeagu, W. (2002). Assessment of the situation and development prospects for cashew nut sector. A report. Nigerian Export Promotion Council, Abuja, Nigeria. Available online at www.unctad.org. 
[2] George, K. E; Rani, J. And Francis, D. J. (1986).Studies on NBR/PVC Blends. Journal of Applied Polymer Science, 32: 2867-2873.

[3] Gyedu- Akoto, E; Oduro, I; Amoah, F. M; Oldham, J. H; Ellis, W. O; Opoku-Ameyaw, K. F. and Rasheed, B. H. (2008). Physico-Chemical Properties of Cashew Tree Gum. African Journal of Food Science, 2: pp. 060-064.

[4] Huang, S. J. (1995). Polymer waste managementbiodegradation, incineration and recycling. J. Macromol. Sci. Pure., 32: 593-597.

[5] Kumar, R; Patil, M. B; Patil, S. R. and Paschapur, M. S. (2009). Evaluation of Anacardium occidentale gum as gelling agent in aceclofenac gel. International Journal of PharmTech Research, 1 (3): 695-704.

[6] Lima, R. D. N., Lima, J. R., de Salis, C. R. and Moreira, R. A. (2002). Cashew-tree (Anacardium occidentale L.) exudate gum: a novel bioligand tool. Biotechnol Appl Biochem. 35: $45-53$.

[7] Mediha, K; Kadir, D._R and Yildirim, A. (2008). Thermophysical Properties of Blend of Poly(Vinyl Chloride) With Poly (Isobornyl Acrylate). International Journal of Science \& Technology, 3 (1): 37-42.
[8] Oh, B. and Kim, Y. R. (1999). Evaluation and characteristics of a blend polymer for a solid polymer electrolyte. Solid state Ionics. 124 (1-2): 83-89.

[9] Pielichowski, K. (1999). Thermal energy storage systems based on poly(vinyl chloride) blends. Eur. Polym. J., 35 (1): 27-34.

[10] Pielichowski, K. and Hamerton, I. (2000). Compatible poly(vinyl chloride)/chlorinated polyurethane blends: thermal characteristics. EurPolym J. 36(1): 171-181.

[11] Rhoo, H. J; Kim, H. T; Park, J. K. and Hwang, T. S. (1997). Ionic conduction in plasticized PVC/PMMA blend polymer electrolytes. Electrochin. Acta, 42(10): 1571-1579.

[12] Scott, G. and Gillead, D. (1995). Degradable Polymers. Chapman \& Hall, London.

[13] Stephen, A. M; Kumar, T. P; Renganathan, N. G; Pitchumani. S; Thirunakaran, R; Muniyandi, N. (2000). Ionic conductivity and FT-IR studies on plasticized PVC/PMMA blend polymer electrolytes. J. Power Sources, 89 (1): 80-87.

[14] Tang, M. and Liau, W. R. (2000). Solvent effect on the miscibility of poly(4- hydroxystyrene)-poly(ethylene oxide) blends. Eur. Polym. J. 36 (12): 2597-2603. 